\title{
Segmental arterial mediolysis in pigs presenting with renal infarcts
}

Leifsson, Páll S.; Slavin, R. E.

Published in:

Veterinary Pathology

DOI:

$10.1177 / 0300985814568360$

Publication date:

2015

Document version

Publisher's PDF, also known as Version of record

Citation for published version (APA):

Leifsson, P. S., \& Slavin, R. E. (2015). Segmental arterial mediolysis in pigs presenting with renal infarcts.

Veterinary Pathology, 52(6), 1157-1162. https://doi.org/10.1177/0300985814568360 


\title{
Segmental Arterial Mediolysis in Pigs Presenting With Renal Infarcts
}

Veterinary Pathology

2015, Vol. 52(6) 1157-1162

(C) The Auchor(s) 2015

Reprints and permission:

sagepub.com/journalsPermissions.nav DOI: $10.1177 / 0300985814568360$

vet.sagepub.com

\author{
P. S. Leifsson' and R. E. Slavin ${ }^{2}$
}

\begin{abstract}
Segmental arterial mediolysis, a rare arteriopathy first reported in humans, is described in the kidneys of 36 pigs slaughtered in an abattoir in Jutland, Denmark. The kidney changes presented themselves macroscopically as one or more cortical wedge-shaped hemorrhagic or pale lesions. The arterial lesions involved the interlobar and arcuate arteries and exhibited injurious and reparative phases of development. Two types of injurious lesions occurred: (I) a tearing separation of the outer media from the adventitia with fibrin, erythrocytes, and edema fluid filling the formed space, causing collapse of the arterial wall, and (2) outer and mid-medial foci showing irregularly bordered cytoplasmic vacuolar change containing membranous and organelle debris or smooth muscle shrinkage with nuclear loss. In the reparative phase, granulation tissue filled and expanded tear sites and zones of arterial medial muscle loss and extended into the adventitia and through the intima into the arterial lumen. Sequelae, including dissecting hematomas and arterial occlusions causing renal infarcts, were found. Although repartitioning agents widely used in animal husbandry in many countries may potentially cause segmental arterial mediolysis, no such link could be identified. The causation of segmental arterial mediolysis in these pigs is currently unknown but is being further investigated.
\end{abstract}

\section{Keywords}

segmental arterial mediolysis, pigs, renal infarcts, dissecting hematomas, vasculopathy, artery

In humans, segmental arterial mediolysis (SAM) is an uncommon noninflammatory disease first recognized in 1976 as a distinct entity occurring in the abdominal arteries of elderly patients. ${ }^{9}$ However, the disease was initially described in 1943 involving the vertebral and posterior inferior cerebellar arteries and in 1949 targeting the coronary arteries of newborns. ${ }^{2,5}$ This entity is a disease of muscular arteries involving the large- and moderate-sized abdominal and retroperitoneal arteries, the extramural coronary arteries, and the arteries at the brain base. It most often presents with catastrophic abdominal hemorrhages, but it may also be introduced as an ischemic disorder caused by the sequelae of repair or transformation to fibromuscular dysplasia. ${ }^{13}$ SAM can also initially be announced by organ injury, mainly renal infarcts or pancreatic hemorrhages. ${ }^{10}$ Finally, this entity may be entirely asymptomatic with radiological resolution of the arterial lesions. ${ }^{10,14}$

On the basis of its morphologic characteristics and initial site of injury at the adventitial-medial junction, it was suspected that SAM was a vasospastic disorder caused by norepinephrine. ${ }^{11}$ Further support for this theory was provided by the discovery of SAM involving the large intrarenal, intrahepatic, and coronary arteries in greyhound dogs administered ractopamine. ${ }^{12,15}$ Ractopamine, a $\beta 2$-adrenergic agonist apparently capable of causing the release of norepinephrine from the peripheral nervous system, is used as a repartitioning agent in animal husbandry for the promotion of muscle growth and suppression of lipogenesis in swine, cattle, and poultry and used illegally for its anabolic effect on striated muscle to enhance athletic performance in racing animals. Indeed, the first report of segmental medial arteriolysis in the veterinary literature followed the necropsy of 2 greyhounds used to investigate the ability of a pari-mutuel laboratory to detect this agent in the urine of racing Greyhounds.

This article is the first to describe unique porcine renal artery lesions causing infarcts, which were serendipitously detected in abattoir specimens evaluated to exclude infectious processes, and show how these alterations are identical to those occurring in human SAM.

\section{Materials and Methods}

Kidneys from 36 pigs slaughtered at an abattoir in Jutland, Denmark, were submitted for pathologic examination by veterinary meat inspection officers because of the presence

\footnotetext{
'Department of Veterinary Disease Biology, University of Copenhagen, Copenhagen, Denmark

${ }^{2}$ Department of Pathology, Legacy Emanuel Hospital and Health Center, Portland, OR, USA

Corresponding Author:

P. S. Leifsson, Department of Veterinary Disease Biology, University of Copenhagen, Ridebanevej 3, DK-1870 Frederiksberg C, Copenhagen, Denmark. Email: ple@sund.ku.dk
} 

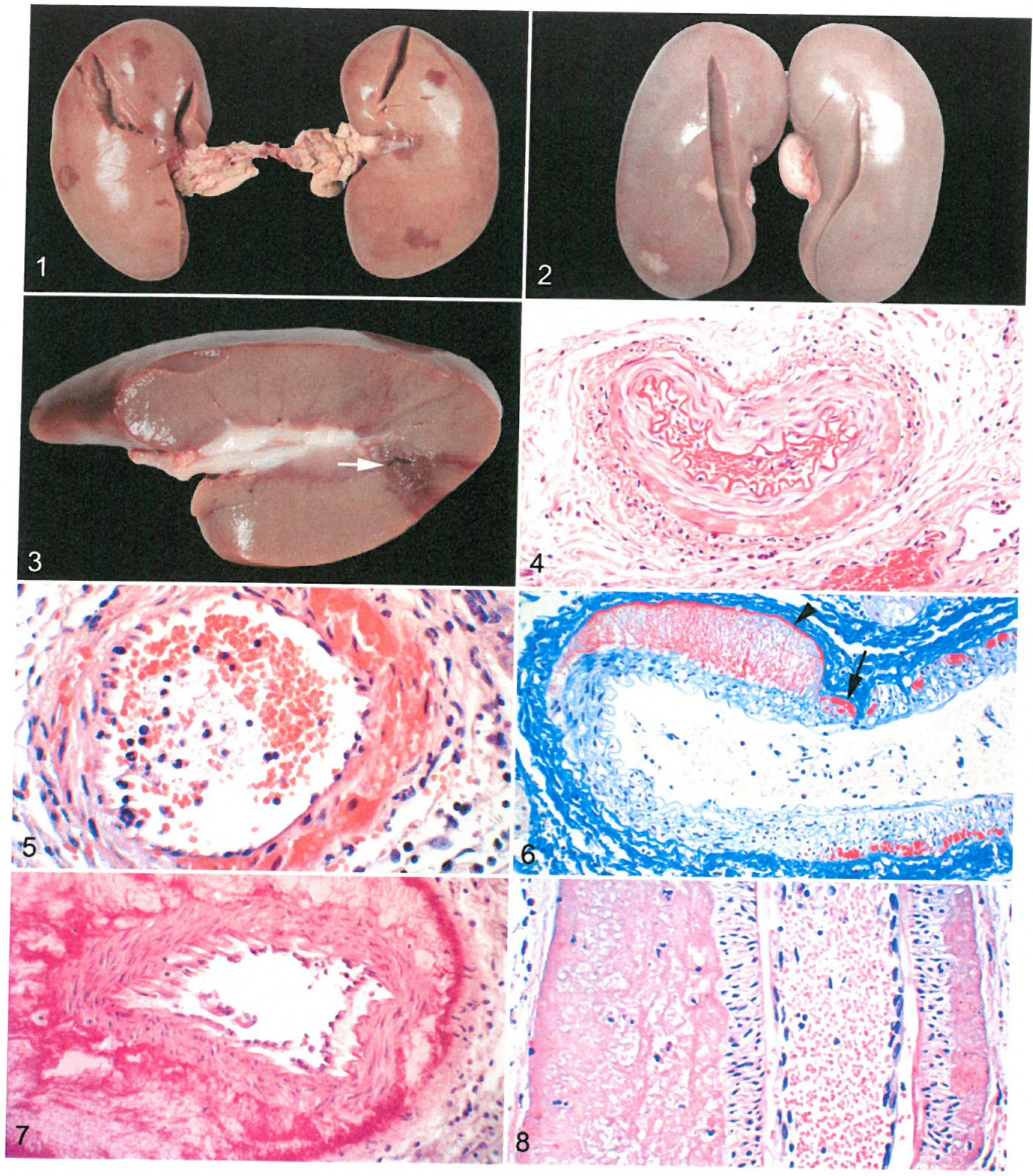

Figures I-3. Infarcts, kidneys, slaughtered pigs. Figure I. Several hemorrhagic infarcts can be seen. Figure 2. Two pale ischemic infarcts are evident in the renal cortex. Figure 3. Cross section shows wedge-shaped hemorrhagic infarct. Tiny linear hematoma (arrow) at the corticomedullary junction at the base of the infarct. Figures 4-10. Injurious phase of segmental arterial mediolysis (SAM), renal arteries, slaughtered pigs. Figure 4. A linear deposit of fibrin can be seen between the adventitia and the media. Hematoxylin and eosin (HE). Figure 5. Microhemorrhages are evident between the adventitia and the media with extension into the media and adventitia. HE. Figure 6. A tearing separation of the adventitia from the outer media. Tear space is expanded by fluid containing fibrin strands that stain red. Its adventitial border is outlined by fibrin (arrowhead). Fibrin deposition is also evident at the adventitial-medial junction bordering the 
of at least 1 cortical pale or hemorrhagic infarct-like lesion without concomitant alterations in other organs. Samples from heart $(n=26)$, liver $(n=26)$, lung $(n=25)$, spleen $(n$ $=21$ ), and mesentery $(n=3)$ were collected from selected pigs. All tissues were fixed in $10 \%$ formalin, processed for histopathology, and stained with hematoxylin and eosin. Selected slides were also stained with Verhoef van Gieson, Martius scarlet blue, Movat pentachrome, and periodic acidSchiff (PAS) reaction.

During a 3-week period, meat-inspecting technicians at the abattoir were instructed to record all cases of pigs with infarctlike lesions fulfilling the criteria listed above with information about the pig's sex and herd of origin.

\section{Results}

\section{Gross Findings}

Gross kidney changes varied from 1 or more cortical wedgeshaped hemorrhagic lesions, many with pale centers, to wedge-shaped lesions that were entirely pale with associated cortical indentations (Figs. 1-3). These lesions were mostly bilateral, but unilateral lesions were also evident mainly in kidneys with a paucity of lesions. In addition, scattered tiny circular or somewhat elongated hemorrhages, 1 to $2 \mathrm{~mm}$ in diameter, were apparent at the base of the lesions or at the corticomedullary junction (Fig. 3).

\section{Histopathology}

The principal alterations in the kidneys were observed in the interlobar and arcuate arteries. The arterial changes were focal and discontinuous. Examination of kidneys from 36 pigs permitted an evaluation of how the renal artery lesions formed and evolved. Injurious and reparative lesions were identified. In the injurious phase, 2 separate arterial lesions developed, one formed at the adventitial-medial junction, the other involving the arterial media. A linear deposit of fibrin (Fig. 4) and/or foci of microhemorrhages (Fig. 5) initiated the adventitial-medial lesion. This was accompanied or followed by a severing of the outer media from the external elastic membrane; a process that could involve part or multiple segments of or almost the entire arterial circumference (Figs. 6, 7). Fibrin, edematous fluid and focal hemorrhages, alone or intermixed to form an eosinophilic coagulum, expanded the tear space (Fig. 8).

Arterial mediolysis was the other injurious lesion. It was less prevalent than the lesions at the adventitial-medial junction but exhibited the same distributional pattern as the latter. This was of two types. In one, the cytoplasm of muscle cells appeared incompletely bordered, vacuolated, and weakly stainable except for the presence of membranous and organelle debris (Fig. 9). In the other type, the smooth muscle cells appeared anucleate and shrunken (Fig. 10). Both degenerative changes principally involved the outer and mid media, but surviving individual muscle cells were often evident in zones of muscle loss and in the inner media. The overlying internal elastic membrane was spared in zones of mediolysis (Fig. 10). Concomitant inflammation was absent. Both injurious lesions could occur together or each could exist separately. Reparative phase changes, characterized by a florid proliferation of granulation tissue, filled and expanded the tear space, frequently pushing the external elastic membrane further away from the media (Figs. 11, 12). It also reoccupied areas of medial loss and in some arteries extended through the internal elastic membrane to bedeck the intima (Fig. 13). The adventitial tissues of involved arteries were also broadened by granulation tissue (Fig. 12). Hemorrhage at the adventitial medial junction or from torn distended capillaries in the granulation tissue in the arterial wall (Fig. 14) separated the outer media from the external elastic membrane to form dissecting hematomas with partial or total collapse of the arterial wall (Figs. 15, 16).

In about half the cases, prominent mesangial hyperplasia was also evident. Parenchymal changes consisted of varying degrees of tubular degeneration and necrosis. In other tissues sampled, no evidence of SAM was detected. Focal chronic inflammatory changes and fibrosis were found in 2 hearts and 2 livers.

\section{Epidemiology}

Epidemiological data collected during the 3-week period proved unreliable and were discarded because of the great variation in diagnostic sensitivity of the abattoir technicians. However, it was their general opinion that the renal lesions were sporadic, although occasionally several cases came from the same herd, especially those delivering many pigs. Furthermore, they agreed that the lesions were seen evenly in males and females.

\section{Discussion}

Noninflammatory arterial changes, mainly fibrinoid necrosis and hyaline degeneration, are seen in pigs with several infectious and noninfectious disorders. ${ }^{6}$ The lesions described here are, however, different both in terms of occurrence, morphology, and location. First, they are found sporadically in otherwise healthy pigs and are not associated with any extrarenal lesions. Second, the shearing of the media from the adventitia described here is not a feature of arterial lesions seen in pigs with circovirus infections, edema disease, or hypovitaminosis E, which are the most important conditions causing arterial lesions in Danish pigs. Third, the lesions described here were exclusively found in the arcuate and interlobar arteries, whereas medium-sized arteries and arterioles are mainly

Figures 4-10. (continued) separation site (arrow). Martius Scarlet Blue. Figure 7. A circumferential separation of the outer media from the adventitia. Separation space is expanded by edema fluid containing clumps and strands of fibrin. Periodic acid-Schiff reaction. Figure $\mathbf{8}$. The space formed by a variably sized circumferential separation is filled with an eosinophilic coagulum. HE. 


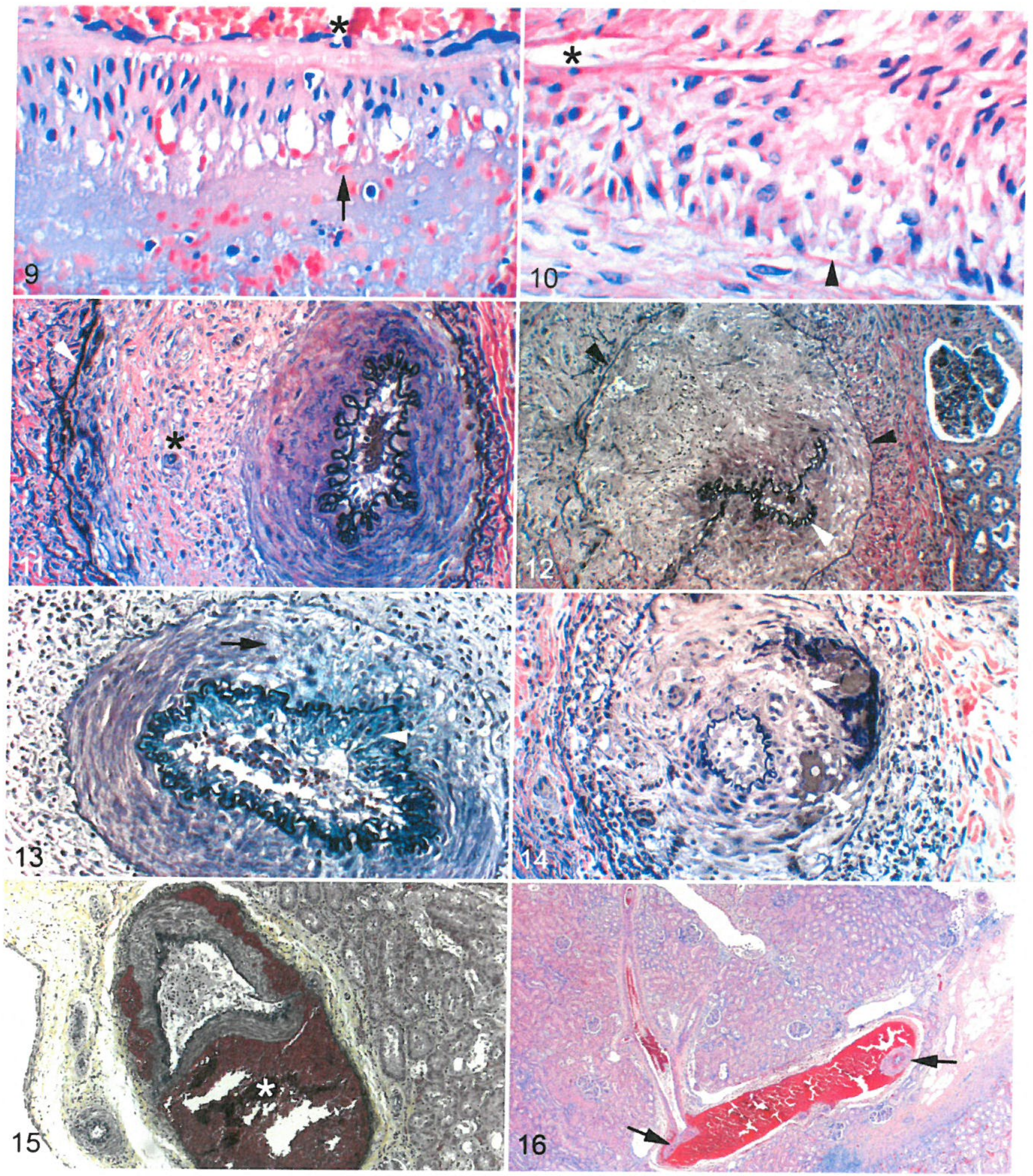

Figure 9. A zone of extensive mediolysis (arrow) principally involves the outer media and abuts a tear space filled with an amphophilic coagulum. Scattered erythrocytes are evident in zones of mediolysis. Asterisk: arterial lumen. HE. Figure 10. Focal loss of smooth muscle cells in the media (arrowhead). The cells show degenerative changes with loss of cell membranes, disintegration of the cytoplasm into eosinophilic granular debris, and nuclear pyknosis and fragmentation. Asterisk: arterial lumen. HE. Figures II-I4. Reparative phase of segmental arterial mediolysis (SAM), kidney arteries, slaughtered pigs. Figure II. Florid proliferation of granulation tissue (asterisk) filling and expanding a separation between outer media and external elastic membrane (arrowhead) with extension into the adventitia. Verhoef van Gieson. Figure 12. Occlusion of the arterial lumen by granulation 
affected in the aforementioned conditions. ${ }^{6,7}$ Therefore, the arterial lesions described in this article are, to our knowledge, unlike any other previously described in pigs, but are they SAM?

In humans, SAM is a biphasic arteriopathy. It was initially identified in elderly patients presenting with catastrophic abdominal hemorrhages that developed from gap aneurysms created from foci of transmural mediolysis with concurrent loss of the internal elastic membrane and overlying intima. ${ }^{13}$ Human SAM can also present as an ischemic disorder alone or with accompanying organ injury in younger adult patients, children, and infants. ${ }^{10,13}$ Significant gap aneurysms do not form in these patients, and their clinical presentation is due to sequelae, including fibromuscular dysplasia, created by the repair of less intense nonsymptomatic arterial lesions formed in the injurious phase of this disorder. ${ }^{13}$ These repaired arterial lesions can also remain totally asymptomatic or resolve. ${ }^{10,14}$

The swine SAM encountered at the abattoir has many of the salient features of nonhemorrhagic SAM. Its 2 injurious lesions described here are identical to those encountered in human SAM, but gaps have not been found. Sequelae developing in swine SAM are identical to those identified in human SAM. ${ }^{10,13,14}$ These include the creation of dissecting hematomas derived not from intimal tears but from hemorrhage at the adventitial-medial junction or in the intramural granulation tissue. The granulation tissue in the tear space, adventitia, and media represents lesions similar to the precursor lesions of human fibromuscular dysplasia, and the proliferation of granulation tissue occluding the arterial lumen is responsible for the ischemic renal lesions grossly identified at the abattoir. Aneurysms, a major sequela encountered in human SAM, were not evident since this lesion arises from arterial gaps, a lesion not detected in the slaughtered pigs. Repairing arterial medial lesions were also evident. Histologically, these were analogous to those encountered in human SAM and explained how SAM could resolve on angiographic studies. ${ }^{10,13}$

The investigation described in this article was prompted by observations made by the veterinary meat inspection officers at an abattoir in Jutland, Denmark. According to meat inspection regulations, carcasses of pigs with recent renal infarcts are considered unfit for human consumption and are discarded. The rationale for this is the strong link between porcine renal infarcts and intravascular infections, mainly endocarditis. ${ }^{4}$ Swine SAM appeared limited to one abattoir in Denmark perhaps because of the increased awareness and a focused study of SAM in this abattoir. It is well known that the incidence of diseases diagnosed at slaughter can be seriously underestimated and varies considerably between abattoirs, although the animals come from the same herds. ${ }^{1,3}$ Because SAM has been associated with ractopamine use, it may be suspected that repartitioning agents are responsible for pig SAM. However, the use of repartitioning agents is prohibited in Denmark, with strict control measures administered by the food safety authorities, which makes the etiological role of these agents unlikely. This is supported by the apparent lack of a relationship of renal SAM to specific herds.

The injurious phase of SAM in humans develops usually in about 1 to 4 days after exposure to the responsible agonist. Since the time interval from leaving the herd to slaughter varies from a few hours to more than a day, the events or agent responsible for the development SAM may have occurred in the herd, in transport, or at the abattoir. We propose that stressful handling or restraint instigated a norepinephrine release at the herds, during transportation, or at the abattoir. This acute stress - the fight-or-flight response-may be the cause of SAM in these pigs. In this response, blood flow from splanchnic organs such as the intestines and kidneys is diverted to the skeletal muscle while the circulation to the heart and brain is unimpaired. The hypothesis that stress caused SAM can be countered by the fact that the arteries of the latter 2 organs are also targeted in human SAM, but their involvement in SAM is putatively determined by arterial $\alpha 1$-adrenergic receptor density fashioned by species and modified by age and sex. ${ }^{8,16}$ It is theoretically possible that the intense stress-related release of norepinephrine may have signaled the high density of $\alpha$-adrenergic receptors in kidneys of select pigs to a nonphysiological pressor response to create SAM in this organ. However, to our knowledge, SAM has never been detected in the fight-orflight response in animals.

In conclusion, this is the first report of spontaneously arising SAM in animals. Many questions are still unanswered about the etiology and pathogenesis of the condition. Further studies are required especially to definitively exclude repartitioning agents as a cause since porcine meat products contaminated by these agents can potentially cause human SAM if eaten. In time, the pig may, hopefully, become a useful model animal for SAM in humans.

\section{Acknowledgements}

We are grateful to Marianne K. Jensen, DVM, Danish Crown, Saeby, Denmark, for providing us with material and information for this

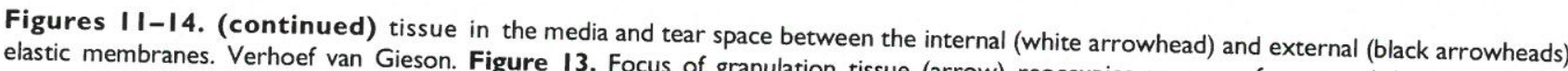
elastic membranes. Verhoef van Gieson. Figure 13. Focus of granulation tissue (arrow) reoccupies an area of transmedial mediolysis and extends inwardly into and over the intima (white arrowhead) through the intact internal elastic membrane (stained black) and outwardly into the Figure 14. Granulation tissue in the media and elastic membrane. Granulation tissue is also evident in the adjacent adventitia. Movat pentachrome. Figure 15. Segmental arterial mediolysis, interlobar artery of kidney, slaughtered pig. A dissecting hematom the outer media and adventitia (yellow). This lesion forms the kidney, slaughtered pig. A dissecting hematoma (asterisk), a sequela, formed between mental arterial mediolysis, arcuate artery of kidney, slaughtered pig. Sequela showing a large dissecting hematomat pentachrome. Figure I6. Segand adventitia. This lesion is responsible for the small linear hemat Sequela showing a large dissecting hematoma formed between the outer media collapsed artery can be seen within the hematoma (arrows). Hematoxylin and eosin. 
study. We thank Betina Andersen, Elizabeth W. Petersen, and Dennis Brok, Department of Veterinary Disease Biology, Faculty of Health and Medical Sciences, University of Copenhagen, for their skillful technical assistance.

\section{Author Contribution}

Conception or design: PL, RS. Data acquisition, analysis, or interpretation: PL, RS. Drafting the manuscript: PL, RS. All authors participated in critically revising the manuscript, gave final approval, and agree to be accountable for all aspects of work to ensure integrity and accuracy.

\section{Declaration of Conflicting Interests}

The author(s) declared no potential conflicts of interest with respect to the research, authorship, and/or publication of this article.

\section{Funding}

The author(s) received no financial support for the research, authorship, and/or publication of this article.

\section{References}

1. Bonde M, Toft N, Thomsen PT, et al. Evaluation of sensitivity and specificity of routine meat inspection of Danish slaughter pigs using latent class analysis. Prev Vet Med. 2010;94:165-169.

2. Gruenwald P. Necrosis in the coronary arteries of newborn infants. Am Heart J. 1949;38:889-897.

3. Jansen ECL, Nielsen AB, Chrisensen KW, et al. Schwannomas-a common finding in slaughter cattle in Denmark. Dansk Veterinaertidsskrift. 2007;90:12-15

4. Karstrup C, Jensen HE, Aalbaek B, et al. Endocarditis-associated brain lesions in slaughter pigs. J Comp Pathol. 2011;144:289-295.
5. Kernohan JW, Woltman HW. Postoperative, focal, nonseptic necrosis of vertebral and cerebellar arteries. J Am Med Assoc. 1943;122:1173-1177.

6. Maxie MG, Robinson WF. Cardiovascular system. In: Maxie MG, ed. Jubb, Kennedy \& Palmer's Pathology of Domestic Animals. Vol 3. 5th ed. Philadelphia, PA: Saunders Elsevier; 2007:56-69.

7. Resendes AR, Segalés J. Characterization of vascular lesions in pigs affected by porcine circovirus type 2-systemic disease [published online June 24, 2014]. Vet Pathol. doi: 10.1177/0300985814540542.

8. Rudner XL, Berkowitz DE, Booth JV, et al. Subtype specific regulation of human vascular alpha-1 adrenergic receptors by vessel bed and age. Circulation. 1999;100:2336-2343.

9. Slavin RE, Gonzales-Vitale JC. Segmental mediolytic arteritis: a clinical pathologic study. Lab Invest. 1976;35:23-29.

10. Slavin RE, Gonzalez JDC, Machin JM, et al. Segmental arterial mediolysis, reparative phase: an analysis and case report showing conversion to fibromuscular dysplasia with renal infarction. World J Cardiovasc Dis. 2014;4: 50-60.

11. Slavin RE, Inada K. Segmental arterial mediolysis with accompanying venous angiopathy: a clinical pathologic review, report of 3 new cases and comments on the role of endothelin-1 in its pathogenesis. Int J Surg Pathol. 2007;15: 121-134.

12. Slavin RE, Yaeger MJ. Segmental arterial mediolysis - an iatrogenic disorder induced by ractopamine. Cardiovasc Pathol. 2012;21:334-338.

13. Slavin RE. Segmental arterial mediolysis: a clinical-pathologic review, its role in fibromuscular dysplasia and differential diagnosis of the masqueradermuscular artery cystic necrosis. World J Cardiovasc Dis. 2013;3:64-81.

14. Slavin RE. Segmental arterial mediolysis: course, sequelae, prognosis, and pathologic-radiologic correlation. Cardiovasc Pathol. 2009;18:352-360.

15. Yaeger MJ, Mullin K, Ensley SM, et al. Myocardial toxicity in a group of greyhounds administered ractopamine. Vet Pathol. 2012;49:569-573.

16. Zhong H, Minneman KP. Alpha-1 adrenoceptor subtypes. Eur J Pharmacol. 1999;375:261-276 